
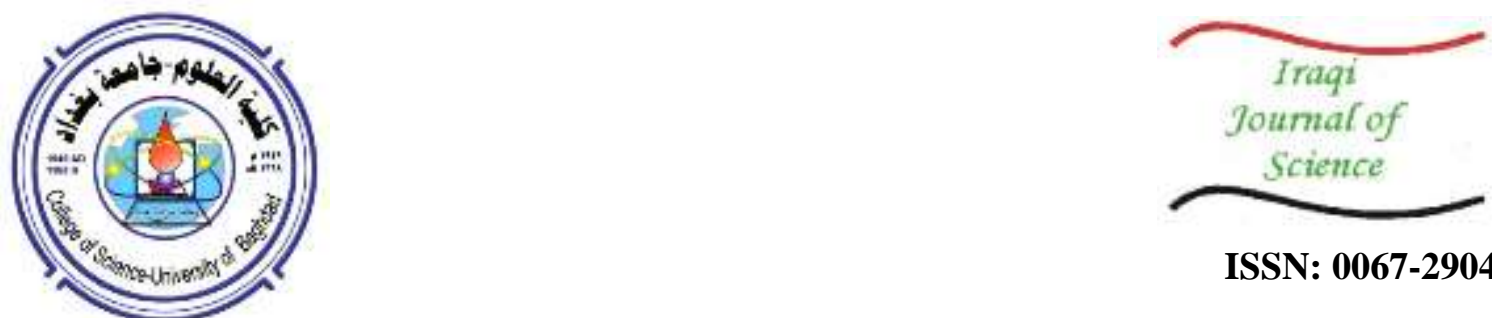

ISSN: 0067-2904

\title{
Peristaltic Transport of Power-Law Fluid in an Elastic Tapered Tube with Variable Cross-Section Induced by Dilating Peristaltic Wave
}

\author{
Mohammed Ali Murad ${ }^{1 *}$, Ahmed M. Abdulhadi ${ }^{2}$ \\ ${ }^{1}$ Department of Mathematics, College of Basic Education, University of Diyala, Diyala, Iraq \\ ${ }^{2}$ Department of Mathematics, College of Science, University of Baghdad, Baghdad, Iraq
}

Received: 20/7/2020

Accepted: 29/9/2020

\begin{abstract}
The peristaltic transport of power-law fluid in an elastic tapered tube with variable cross-section induced by dilating peristaltic wave is studied. The exact solution of the expression for axial velocity, radial velocity, stream function, local shear stress, volume of flow rate and pressure gradient are obtained under the assumption of long wavelength and low Reynolds number. The effects of all parameters that appear in the problem are analyzed through graphs. The results showed that the flux is sinusoidal in nature and it is an increasing function with the increase of $n, k, \phi$ and $F$, whereas it is a decreasing function with the increase of $b$. An opposite behavior for shear strain is noticed compared to pressure gradient. Finally, trapping phenomenon is presented to explain the physical behavior of various parameters. It is noted that the size of the trapping bolus increases with increasing $n, b, \phi$ and $F$ whereas it decreases as $k$ increases. MATHEMATICA software is used to plot all figures.
\end{abstract}

Keywords: Peristaltic transport, dilating peristaltic, power-law fluid, wavelength number

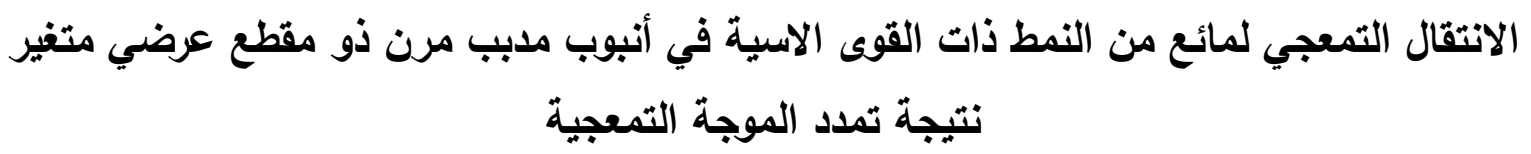

\footnotetext{
2

محمد علي مراد" ، ، احمد مولود عبد الهادي2

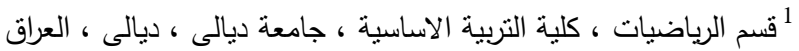

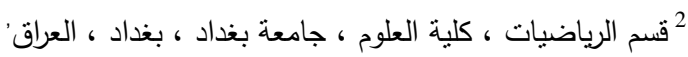

الخلاصة

تمت دراسة الانتقال التمعجي لمائع من النمط ذات القوى الاسية في أنبوب مدبب مرن ذو مقطع عرضي

متغير نتيجة تمدد الموجة التمعجية. تم الحصول على الحل الحقيقي للسرعة المحورية والسرعة الثعاعية ودالة

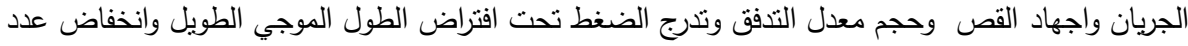

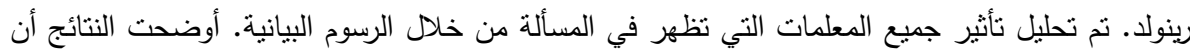

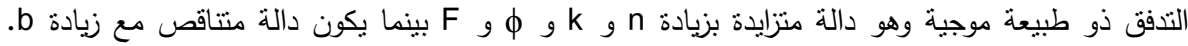

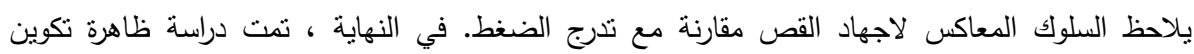

الفقاعات الدائرية بواسطة خطوط الجريان المغلقة عندما يكون الانبوب مستدق لنحليل السلوك الفيزيائي
}

*Email: mindelawy66@yahoo.com 


$$
\begin{aligned}
& \text { لمختلف المعلمات. ولوحظ أن حجم الفقاعات بزداد بزيادة n و و و و F بينما يقل كلما زادت k. تم } \\
& \text { استخدام برنامج MATHEMATICA لرسم جميع الأثكال. }
\end{aligned}
$$

\section{Introduction}

The need to transport fluids through peristaltic channels/tubes has dramatically increased in recent days due partly to its application in engineering and biological system. The peristaltic flow is the transport of fluid generated by wave traveling with invariable speed along the elastic wall in a channel or a tube.

The flow geometry plays an important role to understand the characteristics of different fluid flows. Most of the earlier research works were concentrated on rigid channels. The biological systems deal with elastic boundaries, so the non-Newtonian fluid flow through elastic walls gives some influential applications like swallowing of food though esophagus, blood flow in a small blood vessel, movement of chyme through intestine and colonic vessel, to understand the evolution of pathogen due to vessel deformation. Some of the non-Newtonian fluid models which are accepted by researcher are Jeffery fluid, Herschel-Bulkley fluid, Bingham fluid, power-law fluid etc. The power-law fluid is the simplest and is accepted as a model to understand the rheological properties of physiological fluids in living organisms [1-3].

Srivastava and Srivastava [4] studied the peristaltic transport of a power-law fluid in a uniform and non-uniform channel. The helical flow of a power-law fluid in a thin annulus with permeable walls is discussed by Vajravelu et al. [5]. Pandey and Chaube [6] investigated the peristaltic transport of a visco-elastic fluid in a tube of non-uniform cross section. Sadeghi and Jalali Talab [7] studied the analytical investigation of peristaltic transport of power law fluid through a tube. Hina et al. [8] examined the slip effects on the peristaltic flow of non-Newtonian fluid in a curved channel and found that an intensification in the slip effect resulted in a larger axial velocity. Vajravelu et al. [9] studied the peristaltic transport of a Casson fluid in an elastic tube. Maiti and Pandey [10] analyzed a theoretical study of a nonlinear rheological fluid transport in an axisymmetric tube by cilia. Selvi et al. [11] considered a mathematical model to study the influence of elasticity on the peristaltic flow of a power-law fluid in a tube. Pandey and Singh [12] discussed the peristaltic transport of HerschelBulkley fluids in tubes of variable cross sections induced by dilating peristaltic waves (application to sliding hiatus hernia). The effect of elasticity on peristaltic transport of Herschel-Bulkley fluids in tube of non-uniform cross-section was studied by Selvi and Srinivas [13]. Adnan and Abdulhadi [14] investigated the effect of an inclined magnetic field on peristaltic flow of Bingham plastic fluid in an inclined symmetric channel with slip conditions. Kareem and Abdulhadi [15] studied the impacts of heat and mass transfer on magneto hydrodynamic peristaltic flow having temperature-dependent properties in an inclined channel through porous media. Hasen and Abdulhadi [16] discussed the MHD effect on peristaltic transport for rabinowitsch fluid through a porous medium in cilia channel.

In view of the above studies, a mathematical model is considered to analyze the peristaltic transport of power-law fluid in an elastic tapered tube with variable cross-sections induced by dilating peristaltic wave. The exact expressions for axial velocity, radial velocity, stream function, pressure gradient, local shear stress and volume of flow rate are obtained. The effects of all pertinent parameters on flow are explained through graphs.

\section{Mathematical Formulation}

Consider an axisymmetric peristaltic flow of an incompressible power-law fluid through a cylinder, with variable cross sections, of an elastic tapered tube of length $L$ and radius $H(z)$. The tube walls are subjected to infinite sinusoidal wave with dilating amplitude movement and constant speed $c$, as shown in Figure-1.

In a cylindrical coordinate system $(\bar{R}, \varphi, \bar{Z})$, where $\bar{Z}$ - axis lies along the center line of the tube and $\bar{R}$ is the radius of the tube, at axial station $\bar{Z}$, the instantaneous radius of the tube is given by [12]

$$
\bar{R}=\bar{H}(\bar{Z}, \bar{t}, \bar{k})=a+\bar{b} \bar{Z}-\bar{\emptyset} e^{\bar{k} \bar{Z}} \cos ^{2} \frac{\pi}{\lambda}(\bar{Z}-c \bar{t}),
$$

where $\bar{H}, \bar{Z}, \bar{k}, \bar{t}, a, \bar{b}, \bar{\emptyset}$ and $\lambda$, respectively, denote radial displacement of the wall from the center line, axial coordinate, dilation parameter, time parameter, radius of the tube at the inlet (in the absence of elasticity ), slope of the tube wall (non-uniform parameter), amplitude of the wave, and the wavelength . Because of the axisymmetric condition, the angle $\varphi$ in the cylindrical coordinate is eliminated. 


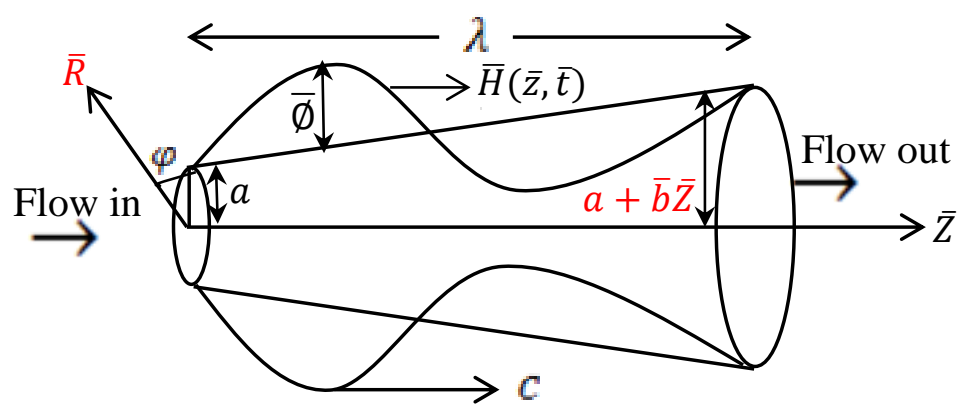

Figure-1 Geometry of the problem

\section{The Basic and Constitutive Equations}

The basic governing equations of non-Newtonian power-law fluid flow are given by: [17] the equation of mass conservation

$$
\nabla \cdot \vec{V}=0 \text {, }
$$

and the equations of momentum conservation (Navier-Stokes equations)

$$
\rho \frac{d \vec{V}}{d t}=\nabla \cdot \overline{\boldsymbol{\sigma}},
$$

in which $\vec{V}$ is the velocity, $\rho$ is the density, $\frac{d}{d t}$ is the material time derivative and $\overline{\boldsymbol{\sigma}}$ is the Cauchy stress tensor.

Let $\vec{V}=(\bar{U}, \bar{V}, \bar{W})$ be the velocity components in the fixed frame cylindrical coordinate system $(\bar{R}, \varphi, \bar{Z})$. According to the assumption of the problem, the velocity components can be written as

$$
\vec{V}=(\bar{U}(\bar{R}, \bar{Z}, \bar{t}), 0, \bar{W}(\bar{R}, \bar{Z}, \bar{t})) .
$$

The power-law fluid model of Ostwald-De Waele is chosen and the constitutive equations can be expressed as: $[11,17]$

$$
\begin{aligned}
& \overline{\boldsymbol{\sigma}}=-\bar{P} I+\bar{\tau}, \\
& \bar{\tau}=-m(\bar{\gamma})^{n-1} \bar{S}, \\
& \bar{S}=2 D,
\end{aligned}
$$

where $\bar{\tau}$ is the extra stress tensor, $I$ is the identity tensor, $\bar{P}$ is the pressure, $\bar{S}$ is the rate of deformation (the rate of strain), $m$ is the consistency parameter (the consistency index of nonNewtonian viscosity), $D=\left\{\frac{1}{2}\left[L+L^{T}\right]: L=\operatorname{grad} \vec{V}\right\}$ is the symmetric part of the velocity gradient (first Rivin-Ericksen tensor), and $\bar{\gamma}$ is defined as:

$$
\bar{\gamma}=\left(\frac{1}{2} \sum_{i} \sum_{j} \bar{S}_{i j} \bar{S}_{i j}\right)^{\frac{1}{2}}=\left(\frac{1}{2} \Gamma_{S_{i j}}\right)^{\frac{1}{2}},
$$

where $\Gamma_{S_{i j}}$ is the second invariant of strain-rate tensor $\bar{S}_{i j}(i, j=1,2,3)$, where the numbers 1,2 and 3 are the coordinates $\bar{R}, \varphi$ and $\bar{Z}$, respectively.

Now, from equations (2) and (3) the continuity and Navier-Stokes equations in the fixed frame are given by

The continuity

$$
\frac{1}{\bar{R}} \frac{\partial(\bar{R} \bar{U})}{\partial \bar{R}}+\frac{\partial \bar{W}}{\partial \bar{Z}}=0 .
$$

The $\bar{R}$ component of Navier-Stokes equations

$\rho\left(\frac{\partial \bar{U}}{\partial \bar{t}}+\bar{U} \frac{\partial \bar{U}}{\partial \bar{R}}+\bar{W} \frac{\partial \bar{U}}{\partial \bar{Z}}\right)=-\frac{\partial \bar{P}}{\partial \bar{R}}-\left[\frac{1}{\bar{R}} \frac{\partial\left(\bar{R} \bar{\tau}_{11}\right)}{\partial \bar{R}}+\frac{\partial \bar{\tau}_{31}}{\partial \bar{Z}}-\frac{\bar{\tau}_{22}}{\bar{R}}\right]$.

The $\bar{Z}$ component of Navier-Stokes equations

$$
\rho\left(\frac{\partial \bar{W}}{\partial \bar{t}}+\bar{U} \frac{\partial \bar{W}}{\partial \bar{R}}+\bar{W} \frac{\partial \bar{W}}{\partial \bar{Z}}\right)=-\frac{\partial \bar{P}}{\partial \bar{Z}}-\left[\frac{1}{\bar{R}} \frac{\partial\left(\bar{R} \bar{\tau}_{13}\right)}{\partial \bar{R}}+\frac{\partial \bar{\tau}_{33}}{\partial \bar{Z}}\right] .
$$

The corresponding boundary conditions are 


$$
\begin{array}{rrr}
\frac{\partial \bar{W}}{\partial \bar{R}}=0 \quad, \quad \bar{U}=0 \quad \text { at } \bar{R}=0 \\
\bar{W}=0 & \text { at } \bar{R}=\bar{H} .
\end{array}
$$

From equation (7), the rate of strain tensor $\bar{S}$ in the fixed frame has the following components [18]

$$
\begin{gathered}
\bar{S}_{11}=2 \frac{\partial \bar{U}}{\partial \bar{R}}, \bar{S}_{22}=2 \frac{\bar{U}}{\bar{R}}, \bar{S}_{33}=2 \frac{\partial \bar{W}}{\partial \bar{Z}}, \\
\bar{S}_{13}=\bar{S}_{31}=\frac{\partial \bar{U}}{\partial \bar{Z}}+\frac{\partial \bar{W}}{\partial \bar{R}}, \quad \bar{S}_{12}=\bar{S}_{21}=\bar{S}_{23}=\bar{S}_{32}=0 .
\end{gathered}
$$

The flow is unsteady in the laboratory frame (fixed frame) and it becomes steady in the wave frame (move frame). The transformation between stationary coordinates $(\bar{R}, \bar{Z})$ and move coordinates $(\bar{r}, \bar{z})$ is given by

$$
\begin{gathered}
\bar{z}=\bar{Z}-c \bar{t}, \quad \bar{r}=\bar{R}, \quad \bar{w}(\bar{r}, \bar{z})=\bar{W}(\bar{R}, \bar{Z}, \bar{t})-c, \\
\bar{u}(\bar{r}, \bar{z})=\bar{U}(\bar{R}, \bar{Z}, \bar{t}), \quad \bar{p}(\bar{r}, \bar{z})=\bar{P}(\bar{R}, \bar{Z}, \bar{t}),
\end{gathered}
$$

where $\bar{u}$ and $\bar{w}$ are the radial and axial velocity components in the moving coordinates.

We introduce the dimensionless quantities that are used to find out the non-dimensional analysis as

$$
\begin{gathered}
\bar{z}=\lambda z, \bar{r}=a r, \bar{u}=\frac{a c u}{\lambda}, \bar{w}=c w, \bar{t}=\frac{\lambda}{c} t, \bar{p}=\frac{c^{n} m \lambda}{a^{n+1}} p, \\
\operatorname{Re}=\frac{\rho a^{n}}{c^{n-2} m}, \bar{\phi}=a \phi, \delta=\frac{a}{\lambda}, \bar{H}=a H, \bar{q}=\pi a^{2} c F, \bar{\gamma}=\frac{c}{a} \gamma, \\
\bar{k}=\frac{k}{\lambda}, \bar{b}=\frac{a}{\lambda} b, \bar{\tau}_{i j}=\frac{c^{n} m}{a^{n}} \tau_{i j} \text { for } i \neq j, \bar{\tau}_{i j}=\frac{c^{n} m}{\lambda a^{n-1}} \tau_{i j} \text { for } i=j, \\
\bar{S}_{i j}=\frac{c}{a} S_{i j} \text { for } i \neq j, \bar{S}_{i j}=\frac{c}{\lambda} S_{i j} \text { for } i=j, \bar{\psi}=c a^{2} \psi,
\end{gathered}
$$

where $\delta$ is the wave number and Re is the Reynolds number (Re $\ll 1) . p, F, \tau_{i j}, S_{i j}$ and $\psi$ are the pressure, the flux, the extra stress tensor, the rate of deformation, and the stream function in nondimensional form, respectively.

Equations (1) and (9)-(13) in the move frame of non-dimensional form are given by:

$$
\begin{gathered}
r=H(z, k)=1+b z-\phi e^{k z} \cos ^{2} \pi z, \\
\frac{1}{r} \frac{\partial(r u)}{\partial r}+\left(\frac{\partial w}{\partial z}\right)=0,
\end{gathered}
$$

$$
\begin{aligned}
& \operatorname{Re} \delta^{3}\left(u \frac{\partial u}{\partial r}+(w+1) \frac{\partial u}{\partial z}\right)= \\
& -\frac{\partial p}{\partial r}-\left[\delta^{2} \frac{1}{r} \frac{\partial\left(r \tau_{11}\right)}{\partial r}+\delta^{2} \frac{\partial \tau_{31}}{\partial z}-\delta^{2} \frac{\tau_{22}}{r}\right] \text {, } \\
& \operatorname{Re} \delta\left(u \frac{\partial w}{\partial r}+(w+1) \frac{\partial w}{\partial z}\right)= \\
& -\frac{\partial p}{\partial z}-\left[\frac{1}{r} \frac{\partial\left(r \tau_{13}\right)}{\partial r}+\delta^{2} \frac{\partial \tau_{33}}{\partial z}\right] \text {, } \\
& \left.\begin{array}{ccc}
\frac{\partial w}{\partial r}=0, \quad u=0 & \text { at } r=0 \\
w=-1 & \text { at } r=H
\end{array}\right\}, \\
& S_{11}=2 \frac{\partial u}{\partial r}, S_{22}=2 \frac{u}{r}, S_{33}=2 \frac{\partial w}{\partial z}, \\
& \left.S_{13}=S_{31}=\delta^{2} \frac{\partial u}{\partial z}+\frac{\partial w}{\partial r}, \quad \bar{S}_{12}=\bar{S}_{21}=\bar{S}_{23}=\bar{S}_{32}=0\right\} \text {. }
\end{aligned}
$$

By applying low Reynolds number and long wavelength approximation (i.e. neglecting wave number), an assumption which is based on the fact that the radius of the tube is small compared to the wavelength of the peristaltic wave, then equations (17)-(19) reduces to

$$
\begin{gathered}
\frac{1}{r} \frac{\partial(r u)}{\partial r}+\left(\frac{\partial w}{\partial z}\right)=0, \\
\frac{\partial P}{\partial r}=0, \\
\frac{\partial p}{\partial z}=-\frac{1}{r} \frac{\partial\left(r \tau_{13}\right)}{\partial r} .
\end{gathered}
$$


The local shear stress can be calculated from equation (6) as follows:

The dimensionless of equation (6), in view of equation (21), is given by

$$
\begin{gathered}
\tau_{13}= \\
-(\gamma)^{n-1}\left(\delta^{2} \frac{\partial u}{\partial z}+\frac{\partial w}{\partial r}\right),
\end{gathered}
$$

Since $\bar{\gamma}=\sqrt{\frac{1}{2} \sum_{i} \sum_{j} \bar{S}_{i j} \bar{S}_{i j}}$ then

$$
\begin{aligned}
& \text { if } i \neq j \text { then } \gamma=\sqrt{\frac{1}{2} \sum_{i} \sum_{j} S_{i j} S_{i j}}, \\
& \text { and if } i=j \text { then } \gamma=\sqrt{\frac{1}{2} \delta^{2} \sum_{i} \sum_{j} S_{i j} S_{i j}} .
\end{aligned}
$$

As mentioned in the previous $\delta \rightarrow 0$ that implies $\gamma=0$ at $i=j$, otherwise substituting equation (21) into equations (26), in view of $\delta \rightarrow 0$, to get $\gamma=\frac{\partial w}{\partial r}$ and the shear rate has been assumed to be negative throughout the moving frame, thus: [3]

$$
\gamma=-\frac{\partial w}{\partial r}
$$

Now, by substituting equation (28) into equation (25), keeping in mind that $\delta \rightarrow 0$, we have

$$
\tau_{13}=\left(-\frac{\partial w}{\partial r}\right)^{n} \text {. }
$$

From equations (24) and (29), we get

$$
\frac{\partial p}{\partial z}=-\frac{1}{r} \frac{\partial\left(r\left(-\frac{\partial w}{\partial r}\right)^{n}\right)}{\partial r} \text {. }
$$

\section{Solution of the Problem}

Rearrange and integrate equation (30) with respect to $r$, in view of equation (23), and apply the first and third boundary conditions of equation (20), then the axial velocity, in view of equation (16), gives

$$
w=-\frac{1}{2^{\frac{1}{n}}\left(\frac{1}{n}+1\right)}\left(-\frac{\partial p}{\partial z}\right)^{\frac{1}{n}}\left(r^{\frac{1}{n}+1}-\left(1+b z-\phi e^{k z} \cos ^{2} \pi z\right)^{\frac{1}{n}+1}\right)
$$

Introducing the axial velocity given in equation (31) into continuity equation (22) and solving it together with the second boundary condition of equation (20) and then dividing the result by $r$, introduces the radial velocity as

$$
\begin{aligned}
u= & \frac{1}{2^{\frac{1}{n}+1}\left(\frac{1}{n}+1\right)} \frac{1}{n}\left(-\frac{\partial p}{\partial z}\right)^{\frac{1}{n}-1} \frac{\partial^{2} p}{\partial z^{2}}\left(1+b z-\phi e^{k z} \cos ^{2} \pi z\right)^{\frac{1}{n}+1} r- \\
& \frac{1}{2^{\frac{1}{n}}\left(\frac{1}{n}+1\right)\left(\frac{1}{n}+3\right)} \frac{1}{n}\left(-\frac{\partial p}{\partial z}\right)^{\frac{1}{n}-1} \frac{\partial^{2} p}{\partial z^{2}} r^{\frac{1}{n}+2}-\frac{1}{2^{\frac{1}{n}+1}}\left(-\frac{\partial p}{\partial z}\right)^{\frac{1}{n}} * \\
& \quad\left(1+b z-\phi e^{k z} \cos ^{2} \pi z\right)^{\frac{1}{n}}\left(b-\phi k e^{k z} \cos ^{2} \pi z+\pi \phi e^{k z} \sin 2 \pi z\right) r .
\end{aligned}
$$

The relationship between the stream function and the velocity components is

$$
w=\frac{1}{r} \frac{\partial \psi}{\partial r} \quad, u=-\frac{1}{r} \frac{\partial \psi}{\partial z} .
$$

By using the conditions ( $\psi=0$ at $r=0)$ and from equations (31) and (33), the stream function is defined as

$$
\psi=-\frac{1}{2^{\frac{1}{n}}\left(\frac{1}{n}+1\right)}\left(-\frac{\partial p}{\partial z}\right)^{\frac{1}{n}}\left(\frac{1}{\frac{1}{n}+3} r^{\frac{1}{n}+3}-\left(1+b z-\phi e^{k z} \cos ^{2} \pi z\right)^{\frac{1}{n}+1} \frac{r^{2}}{2}\right)-\frac{r^{2}}{2} .
$$

The volume flux $\bar{q}$ through any cross-section is given by

$$
\bar{q}=2 \pi \int_{0}^{\bar{H}} \bar{w} \bar{r} d \bar{r},
$$

and the non-dimensional volume flux $F$ is given by 


$$
F=2 \int_{0}^{H} w r d r
$$

Applying equation (31) into equation (35), gives

$$
F=\frac{1}{2^{\frac{1}{n}}\left(\frac{1}{n}+3\right)}\left(-\frac{\partial p}{\partial z}\right)^{\frac{1}{n}}\left(1+b z-\phi e^{k z} \cos ^{2} \pi z\right)^{\frac{1}{n}+3}-\left(1+b z-\phi e^{k z} \cos ^{2} \pi z\right)^{2} .
$$

Solving equation (36) for $\frac{\partial p}{\partial z}$, yields the pressure gradient as

$$
\frac{\partial p}{\partial z}=\frac{-2\left(\frac{1}{n}+3\right)^{n}\left(F+\left(1+b z-\phi e^{k z} \cos ^{2} \pi z\right)^{2}\right)^{n}}{\left(1+b z-\phi e^{k z} \cos ^{2} \pi z\right)^{3 n+1}}
$$

\section{Result and Discussion}

This section deals with the computational results of the peristaltic transport of non-Newtonian power-law fluid in an elastic tapered tube with variable cross sections induced by dilating peristaltic wave. The effect of various parameters that appear in the problem, such as power-law index $n$, slope of the tube wall (non-uniform parameter) $b$, dilation parameter $k$ and amplitude ratio $\phi$, on the axial velocity $w$, radial velocity $u$, pressure gradient $\frac{\partial P}{\partial z}$, volume flux $F$, local shear stress $\tau_{13}$, and trapping of the flow, are discussed graphically. MATHEMATICA software is used to plot all figures.

\subsection{Axial Velocity $w$}

The variation of axial velocity along the radial direction at $z=0.1$ for different values of $n, k, \phi$ and $b$ are analyzed in Figures- -6 , respectively. Note that the axial velocity exhibits an oscillating behavior with the increase of $n, k$ and $\phi$, as shown in Figures-2 - 4. It is observed that an increase in $n, k$ and $\phi$ leads to an increase in the axial velocity at the center of the tube along with a decrease near the boundary. Also, as shown in Figure-5, the non-uniform parameter $b$ exerts the greatest impact on the axial velocity. It is found that, at the center of the tube, the axial velocity decreased with increasing $b$ while it is increasing at the boundary. The axial velocity increased with increasing $F$, as explained in Figure-6.

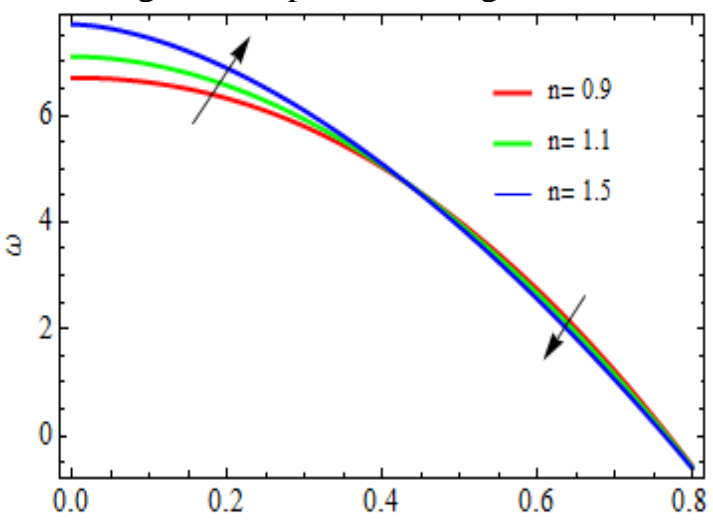

Figure 2- Effect of $n$ on the axial velocity $\omega$ at

$$
k=0.1, b=0.05, \phi=0.2, F=2, z=
$$
0.1

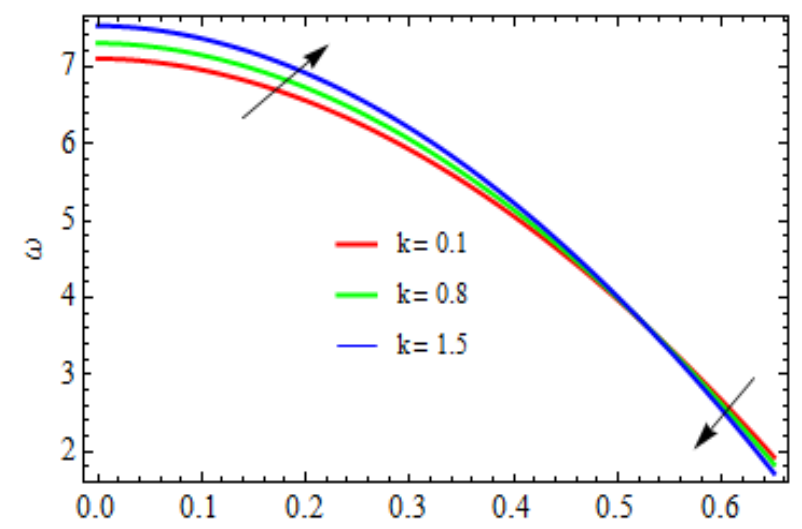

Figure 3- Effect of $k$ on the axial velocity $\omega$ at $n=1.1, b=0.05, \phi=0.2, F=2, z=$ 0.1 


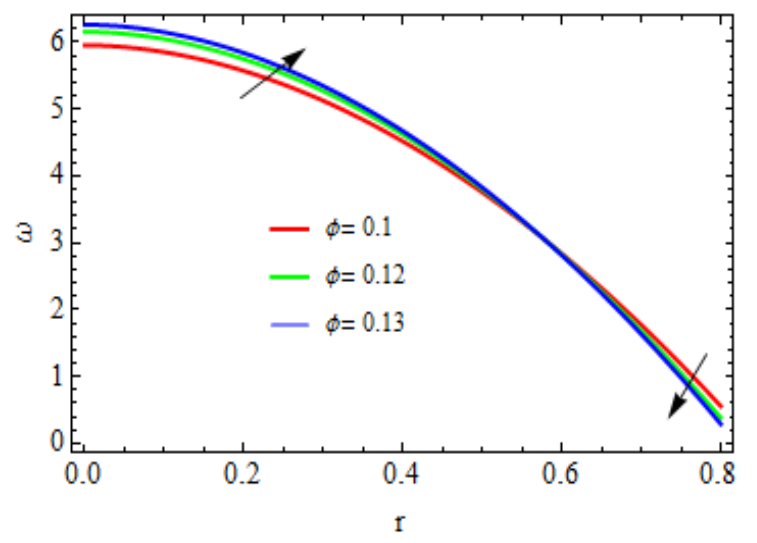

Figure 4- Effect of $\phi$ on the axial velocity $\omega$ at $n=1.1, b=0.05, k=0.2, F=2, z=0.1$

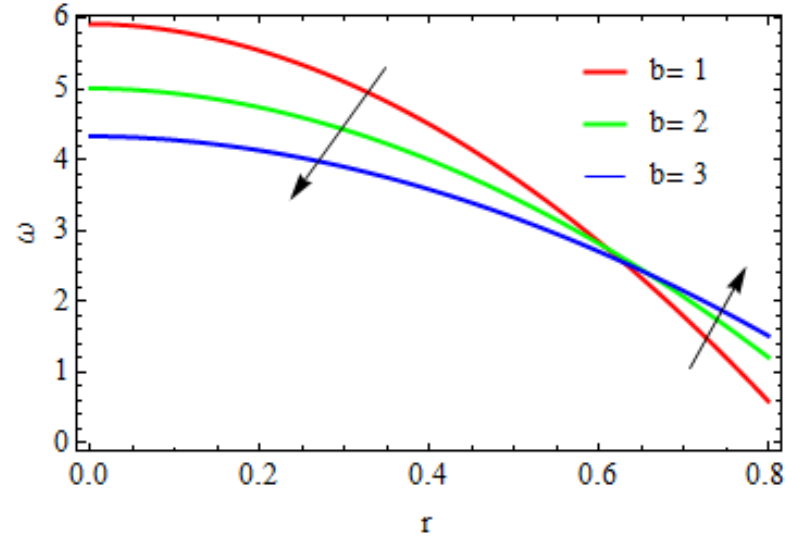

Figure 5- Effect of $b$ on the axial velocity $\omega$ at $n=1.1, k=0.1, \phi=0.2, F=2$, $z=0.1$

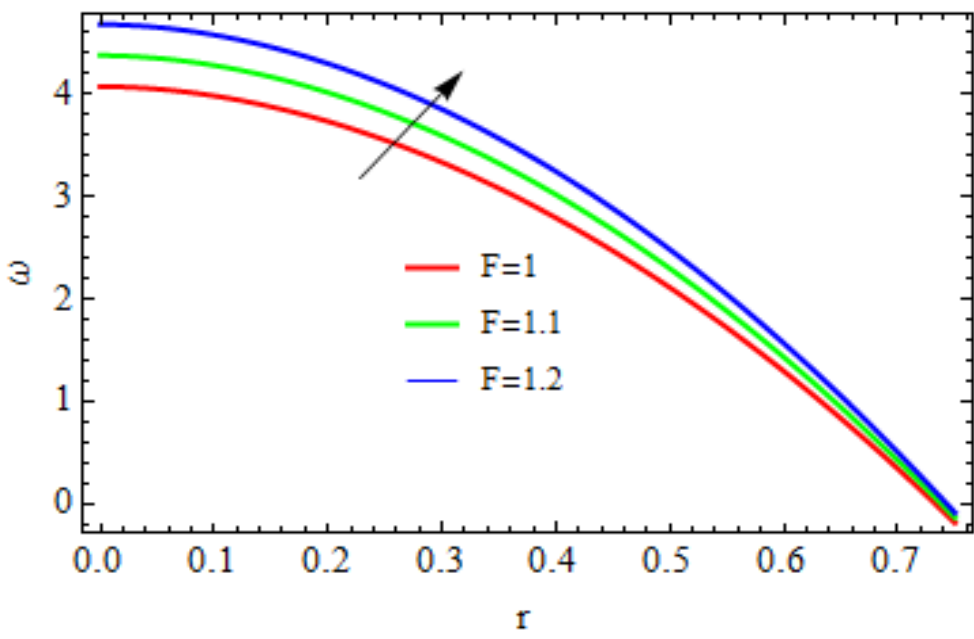

Figure 6- Effect of $F$ on the axial velocity $\omega$ at $n=1.1, b=0.05, k=0.1, \phi=0.2, \quad z=$

0.1

\subsection{Radial Velocity $u$}

Figures 7-10 present the behavior of various parameters involved in the radial velocity $u$ along the radial direction. Figure -7 shows the effect of power law index $n$ on radial velocity. It is clear that the radial velocity reduces with an increase in $n$. Figure -8 elucidates the effect of $\phi$ on the radial velocity. It is noted that an increase in $\phi$ causes the greatest decreases in the radial velocity. The increase in amplitude ratio $\phi$ means a powerful peristaltic movement effect, which causes faster movement in the radial direction for the radial velocity. The impact of the non-uniform parameter $b$ on radial velocity is shown in Figure -9. It is observed that radial velocity increases with increasing $b$. An opposite trend is observed in the case of $k$, as shown in Figure -10 . 


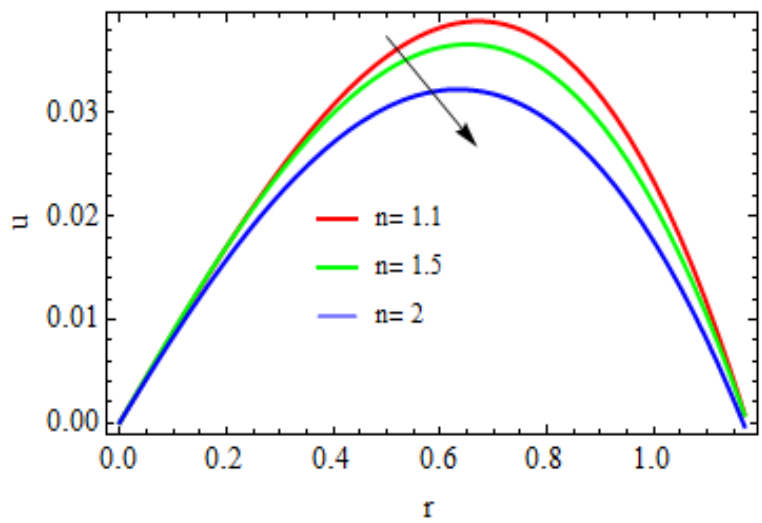

Figure 7- Effect of $n$ on the radial velocity $u$ at $b=0.05, k=0.1, \phi=0.2, p=1$, $7=$ ?

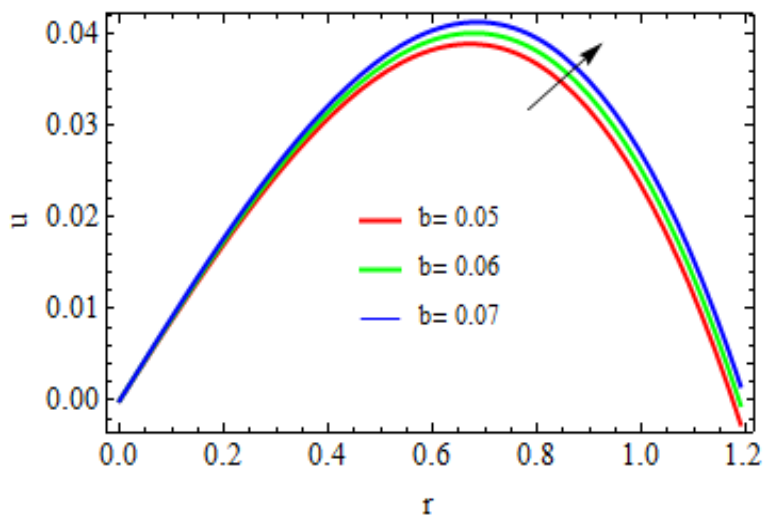

Figure 9- Effect of $b$ on the radial velocity $u$ at $n=1.1, k=0.1, \phi=0.2, p=1$, $z=2$

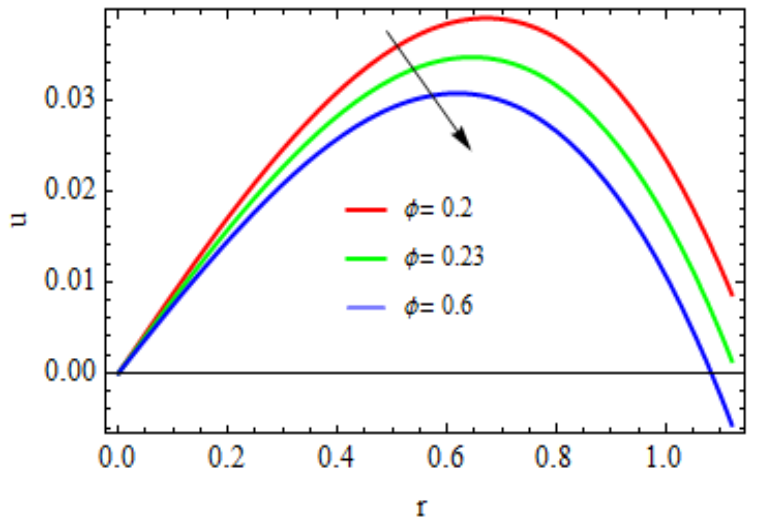

Figure 8- Effect of $\phi$ on the radial velocity $u$ at $n=1.1, b=0.05, k=0.1, p=1, z=$ 2

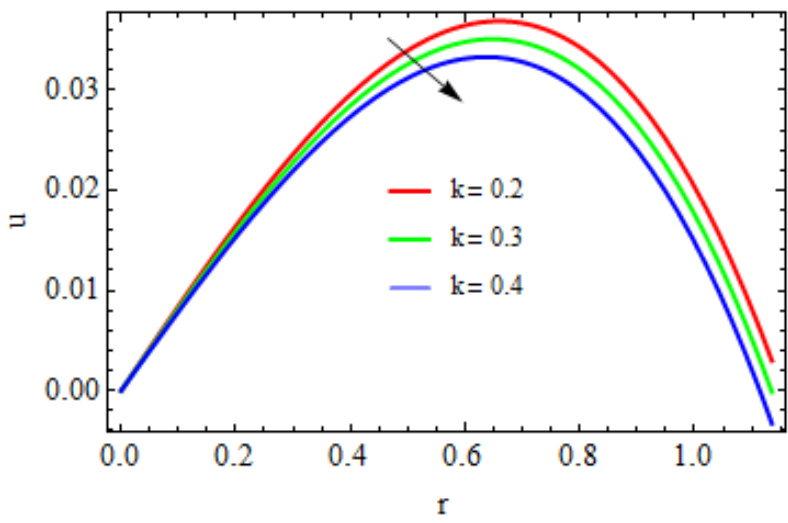

Figure 10- Effect of $k$ on the radial velocity $u$ at $n=1.1, \quad b=0.05, \phi=$ $0.2, p=1, z=2$

\subsection{Volume of Flow Rate (Flux) $F$}

In this subsection, the variation of flux $F$ for different values of parameters, such as power-law index $n$, non-uniform parameter $b$, dilation parameter $k$, and amplitude ratio $\phi$, are discussed. Figures $11-15$ show that the flux along non-uniform tube radius for different values of pertinent parameters are sinusoidal in nature. Figure -11 illustrates the effect of power-law index $n$ on the flux. It is noticed that the flux in an elastic tube increases with the increase in $n$. This result is in conformity with the result in Sadeghi and Jalali Talab [7]. The influence of non-uniform parameter $b$ on flux variation is explained in Figure -12. It is found that the flow rate decreases with the increase in $b$. The opposite behavior is observed in the case of dilation parameter $k$, as shown in Figure -13. The effect of amplitude ratio $\phi$ on flux variation is described in Figure -14. It is clear that the flow rate enhances for increasing values of $\phi$, due to an increase in maximum displacement of the fluid particles. Hence, the increases in fluid velocity yields an enhanced flow rate. This result is in agreement with the result obtained by Selvi et al. [9]. Figure -15 shows that the flux increases with the increase in the pressure gradient. 


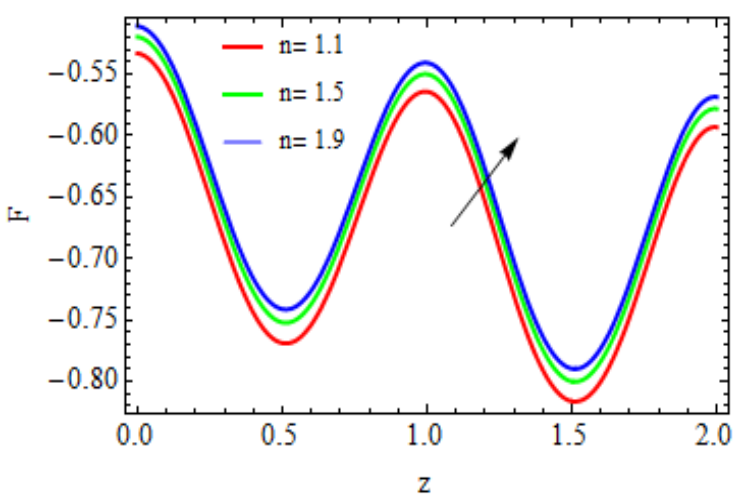

Figure 11- Effect of $n$ on the volume flow rate $F$ at $b=0.05, k=0.1, \phi=$ $0.2, p=2$

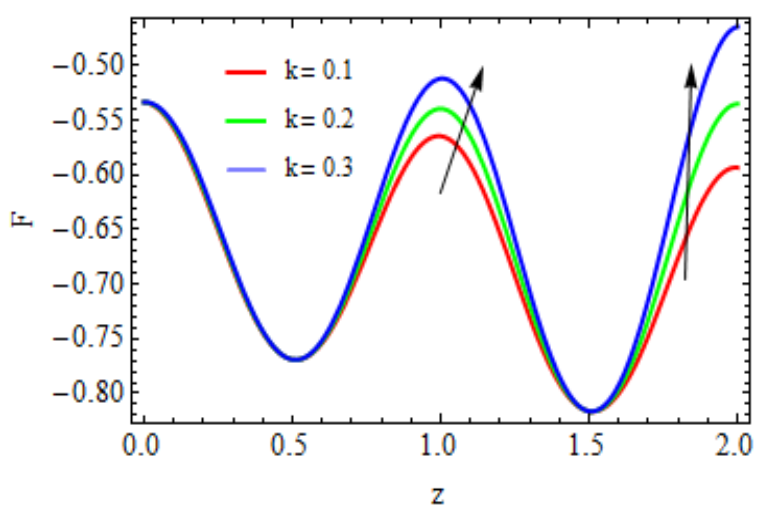

Figure 13- Effect of $k$ on the volume flow rate $F$ at $n=1.1, b=0.05, \phi=0.2, p=2$

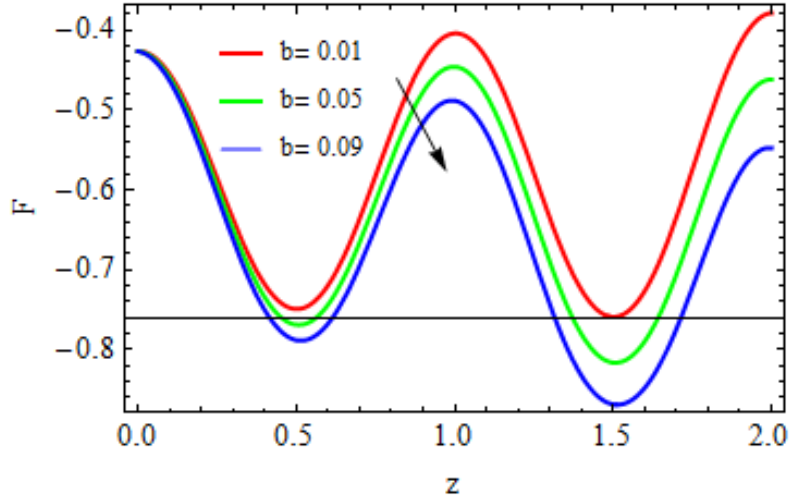

Figure 12- Effect of $b$ on the volume flow rate $F$ at $n=1.1, k=0.1, \phi=0.2, p=$ 2

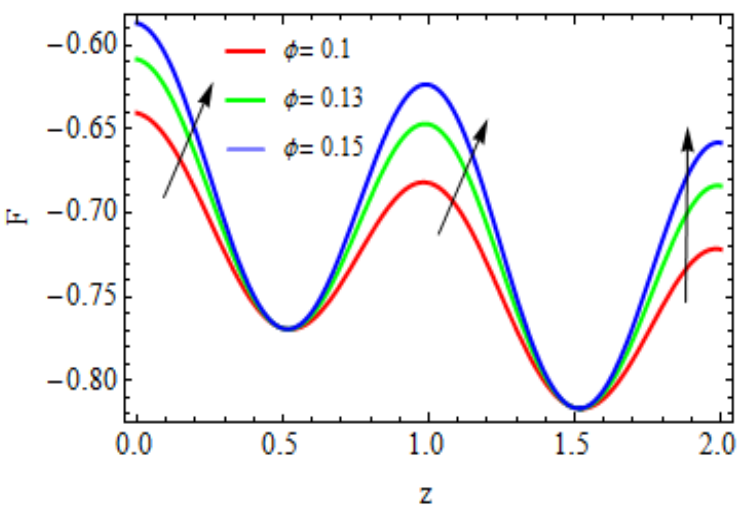

Figure 14- Effect of $\phi$ on the volume flow rate $F$ at $n=1.1, b=0.05, k=$ $0.1, p=2$

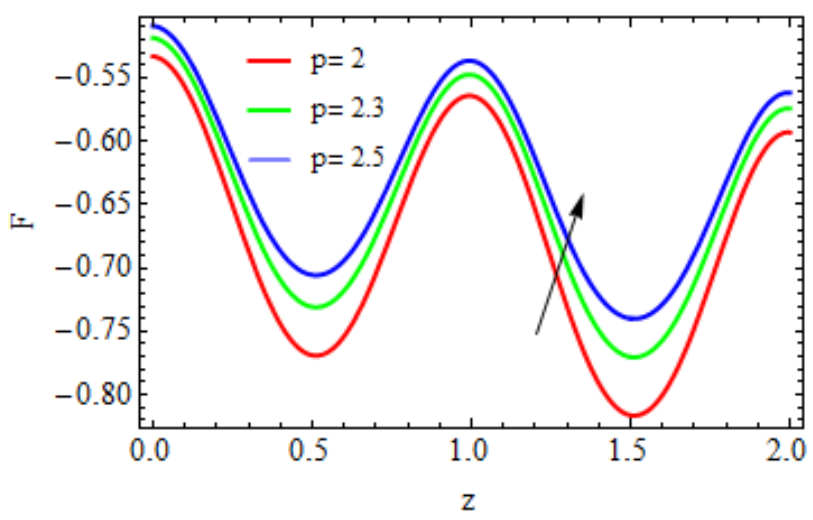

Figure 15- Effect of $p$ on the volume flow rate $F$ at $n=1.1, b=0.05, k=0.1, \phi=0.2$

\subsection{Pressure Gradient $\frac{\partial p}{\partial z}$}

The influence of physical parameters on pressure gradient $\frac{\partial p}{\partial z}$ is represented in Figures $16-20$. As a result of peristaltic property of the tube wall, it may expand or contract. Due to this property of the tube wall, there exist changes in the shape of cross-section of the tube. It can be noticed that, in the wider part of the tube $\mathrm{z} \in[0,0.2]$ and $[0.8,1]$, the pressure is relatively small, that is, the flow is easily passing throughout the tube. On the other hand, in a narrow part of the tube $\mathrm{z} \in[0.2,0.8]$, a much higher pressure is required to maintain the same flux to pass it, especially at approximately $\mathrm{z}=$ 
0.5.The impact of fluid behavior index $n$ on pressure gradient is illustrated in Figure -16 and it is clear that the pressure gradient is reduced with increasing $n$. The effect of the non-uniform parameter $b$ on $\frac{\partial p}{\partial z}$ is shown in Figure -17 . It is observed that $\frac{\partial p}{\partial z}$ increase with the increase in $b$. Figure -18 displays the impact of the dilation parameter $k$ on pressure gradient, where it is noted that the pressure gradient decreases with the increase of $k$. Figures -19 and 20 show that pressure gradient is inversely proportional to the increase of the amplitude of the wave $\phi$ and rate of flux .

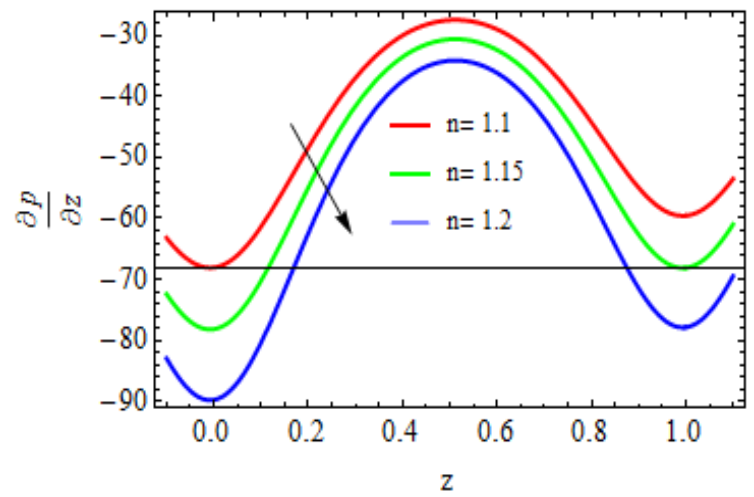

Figure 16- Effect of $n$ on the pressure gradient $\frac{\partial p}{\partial z}$ at $b=0.05, k=0.1, \phi=0.2, F=2$

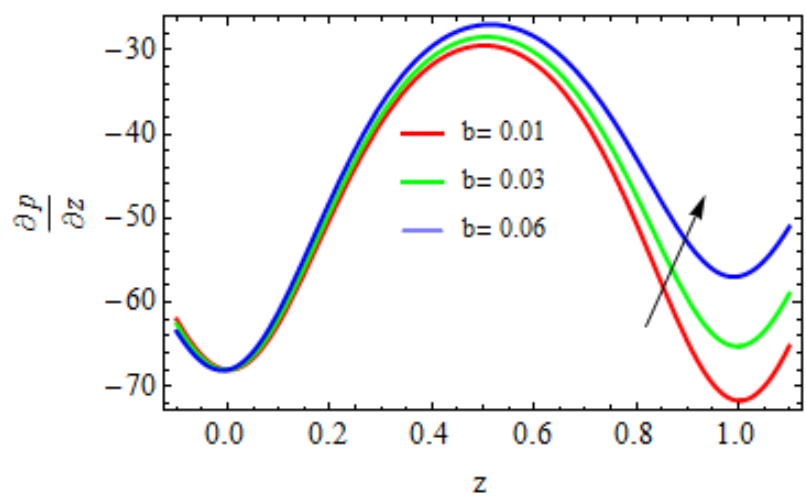

Figure 17- Effect of $b$ on the pressure gradient $\frac{\partial p}{\partial z}$ at $n=1.1, k=0.1, \phi=$ $0.2, F=2$

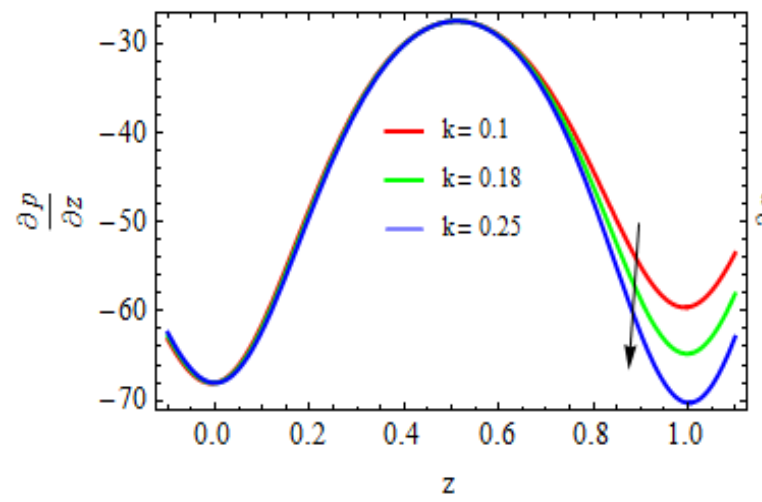

Figure 18- Effect of $k$ on the pressure gradient $\frac{\partial p}{\partial z}$ at $n=1.1, b=0.05, \phi=0.2$, $F=2$

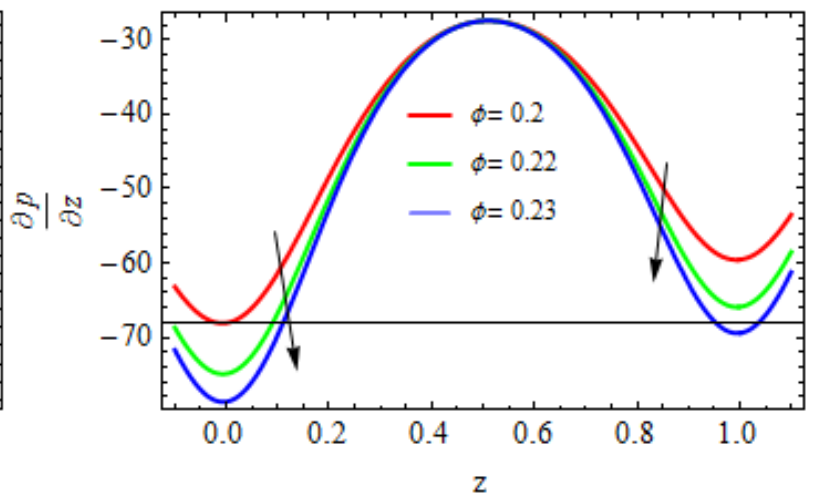

Figure 19- Effect of $\phi$ on the pressure gradient $\frac{\partial p}{\partial z}$ at $n=1.1, b=0.05, k=0.1$, $F=2$

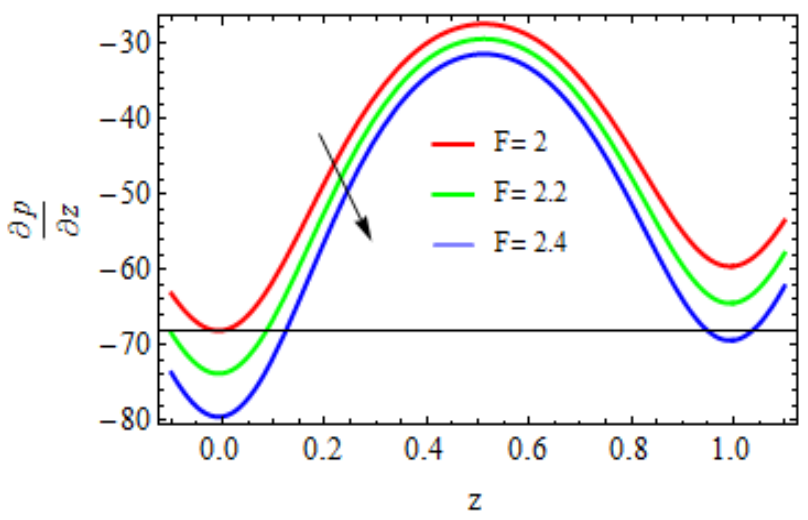

Figure 20- Effect of $F$ on the pressure gradient $\frac{\partial p}{\partial z}$ at $n=1.1, b=0.05, k=0.1, \phi=0.2$ 


\subsection{Shear Stress $\tau_{13}$}

The behavior of shear stress $\tau_{13}$ at the wall of the tube (i.e. at $r=H$ ) for the variation of different parameters is illustrated in Figures $21-25$. It can be observed from Figures $21-24$ that the shear stress increases with the increase of $n, k, \phi$, and $F$. Figure -25 shows that the shear stress decreases with the increase of the non-uniform parameter $b$.

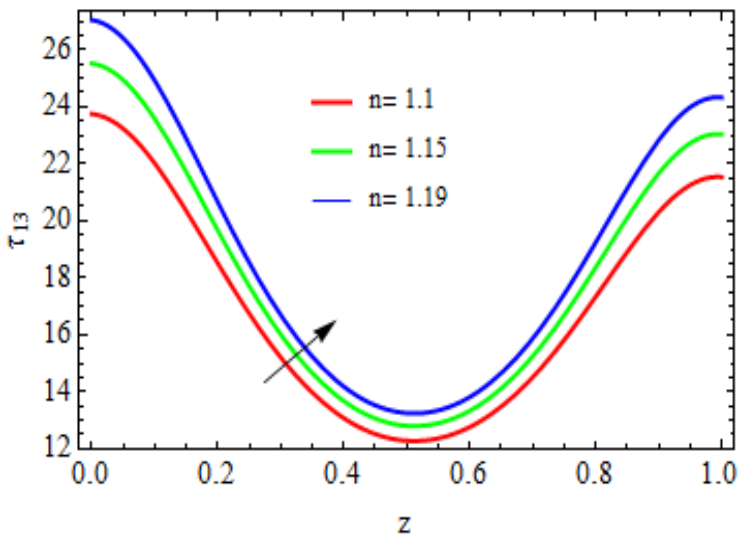

Figure 21- Effect of $n$ on the shear stress $\tau_{13}$ at $b=0.05, k=0.1, \phi=0.2$, $F=2$

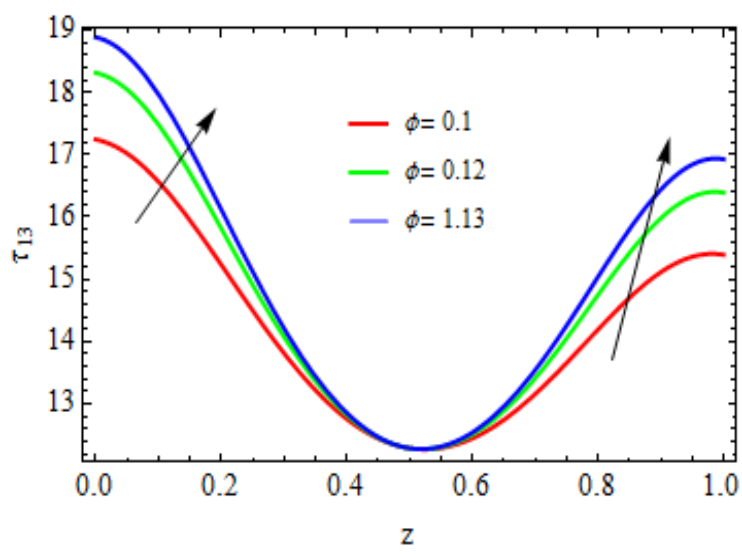

Figure 23- Effect of $\phi$ on the shear stress $\tau_{13}$ at $n=1.1, b=0.05, k=0.1$, $F=2$

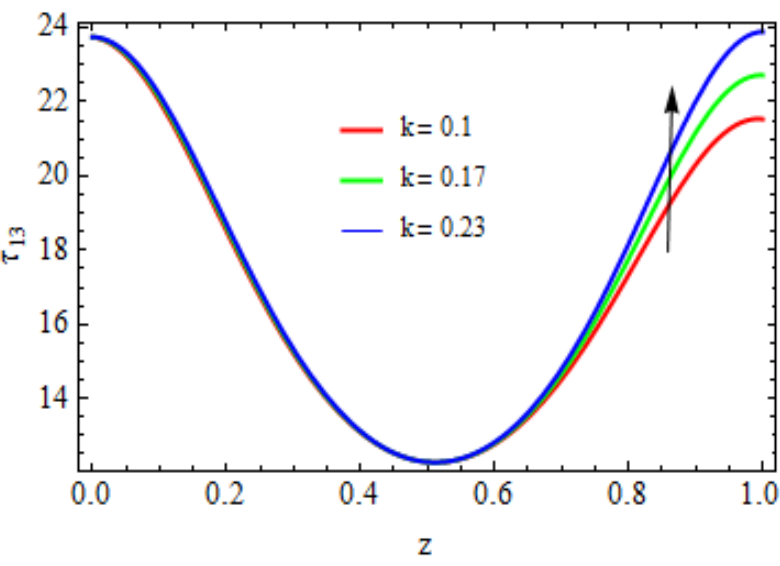

Figure 22- Effect of $k$ on the shear stress $\tau_{13}$ at $n=1.1, b=0.05, \phi=0.2, F=2$

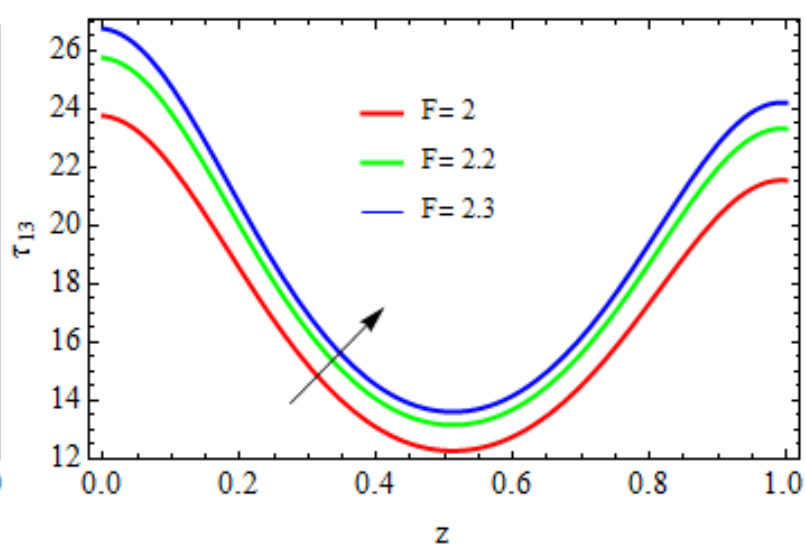

Figure 24- Effect of $F$ on the shear stress $\tau_{13}$ at $n=1.1, b=0.05, k=0.1$, $\phi=0.2$

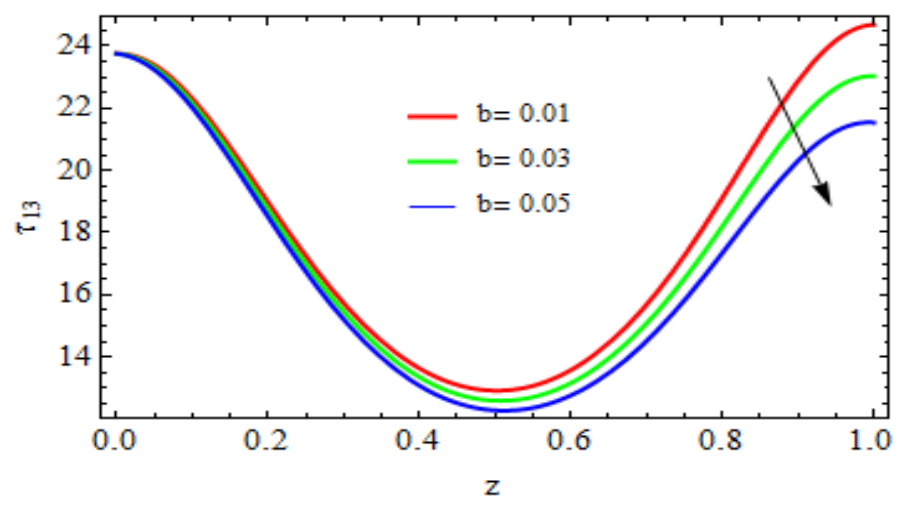

Figure 25- Effect of $b$ on the shear stress $\tau_{13}$ at $n=1.1, k=0.1, \phi=0.2, F=2$ 


\subsection{Trapping Phenomenon}

The formation of an internally circulation bolus of fluid by the closed streamlines is known as trapping. Trapping is an interesting phenomenon of peristalsis mechanism in the wave frame which is pushed ahead with the peristaltic wave. The influences of different parameters on the size of trapped bolus are shown in Figures 26 - 30. It is noted from Figure -26 that the size of the trapped bolus increases as the power-law index $n$ increases. This result is in agreement with the result obtained by Selvi et al. [9]. The impact of the non-uniform parameter $b$ is explained in Figure -27. It is observed that the trapped bolus increases with increasing $b$. It is clear from Figure -28 that the bolus size is reduced with increasing values of dilation parameter $k$. The bolus size enhances with increasing values of amplitude ratio $\phi$, as illustrated in Figure -29. This result is in agreement with the result obtained by Selvi et al. [9] and Selvi and Srinivas [11]. Figure -30 displays the effect of increasing flux on streamlines. It is noted that the number and size of the trapped bolus increases.
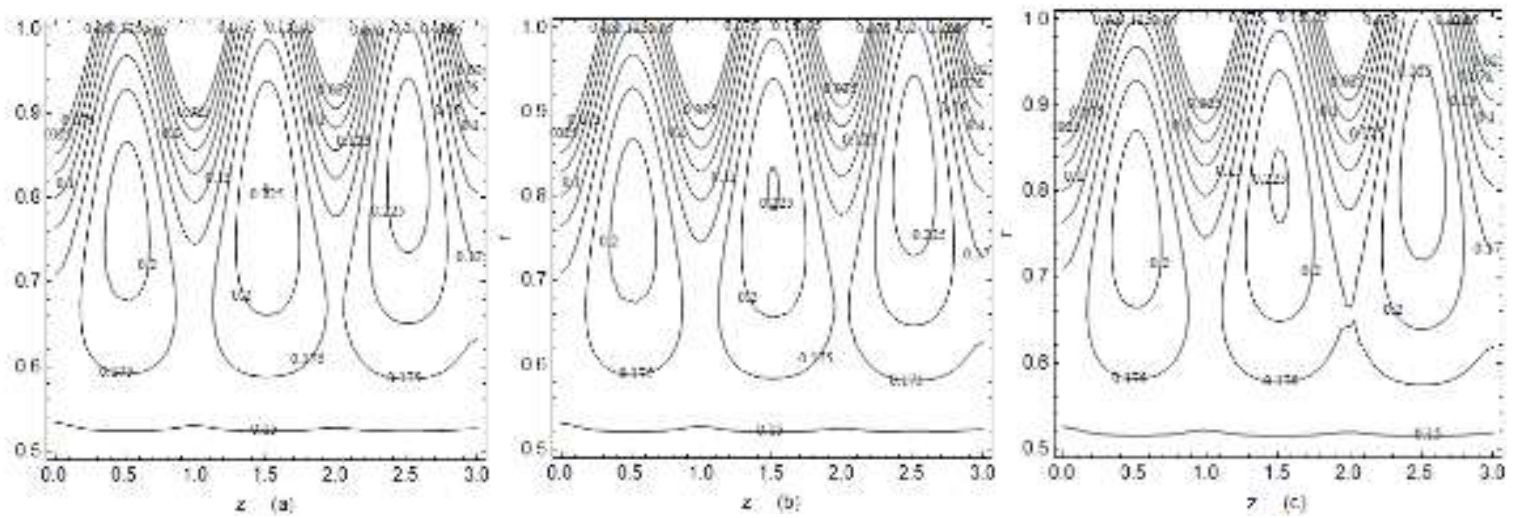

Figure 26- Effect of (a) $n=1.1,(b) n=1.15,(c) n=1.25$ on the stream line at $b=0.05, k=0.1, \phi=0.2, F=0.2$
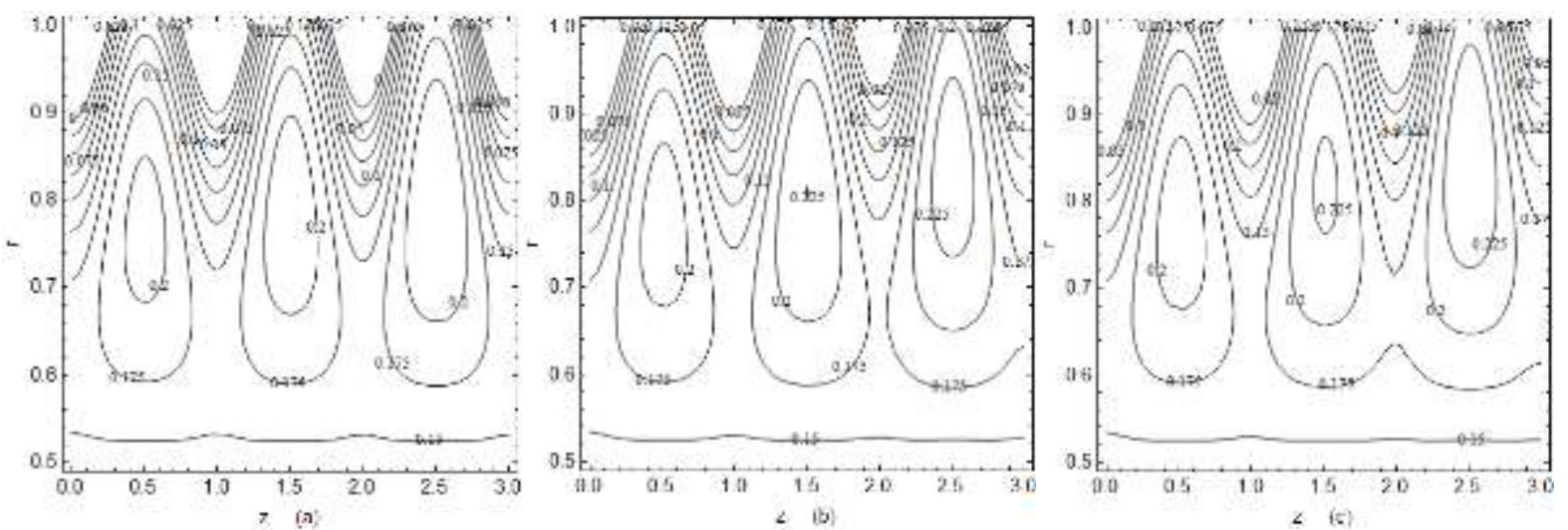

Figure 27- Effect of $(a) b=0.03,(b) b=0.05,(c) b=0.06$ on the stream line at $n=$ 1.1, $k=0.1, \phi=0.2, F=0.2$
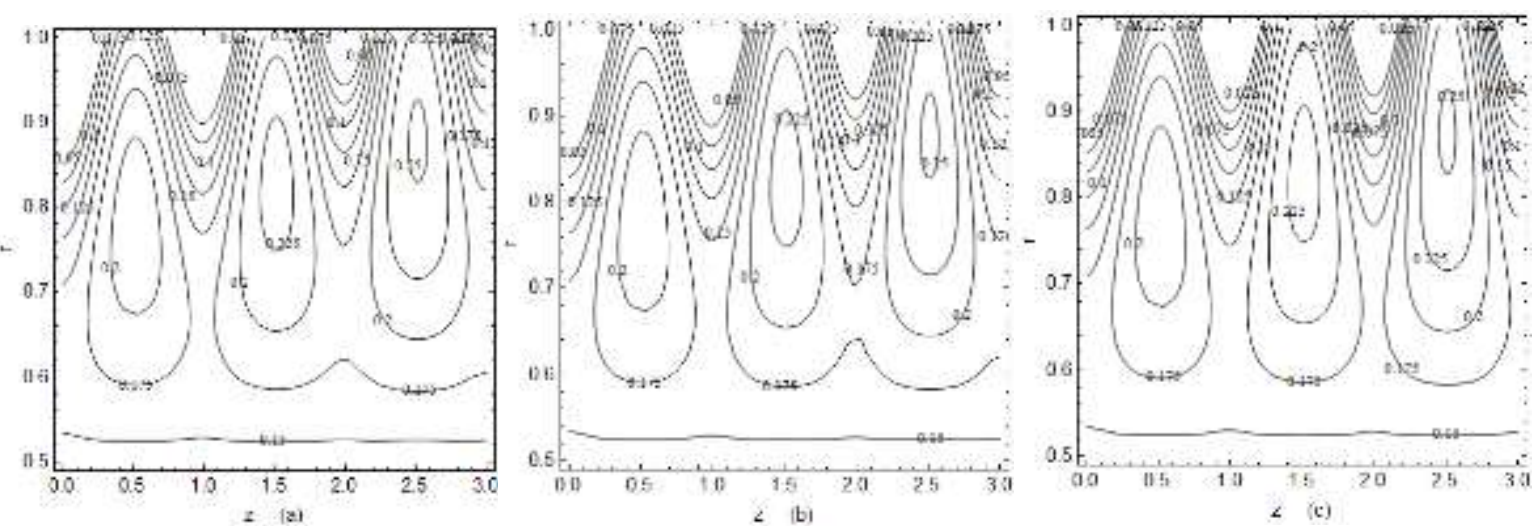

Figure 28- Effect of $(a) k=0.1,(b) k=0.15,(c) k=0.19$ on the stream line at $n=1.1$, $b=0.05 . \phi 13040.2 . F=0.2$ 

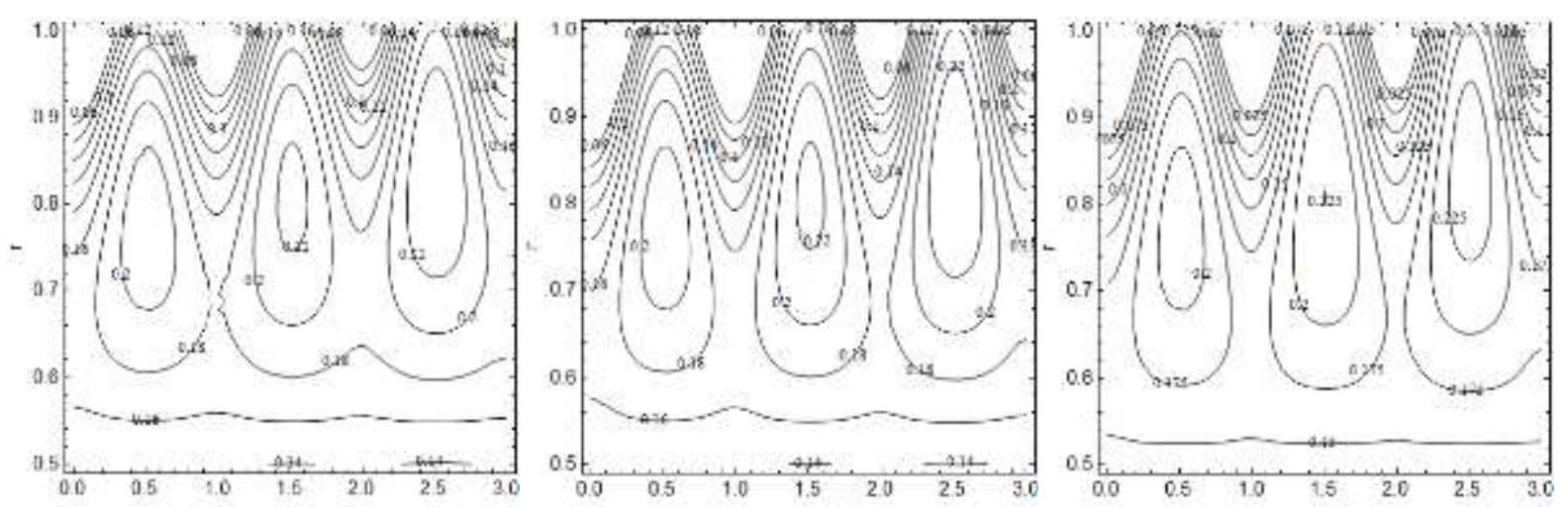

Figure 29- Effect of $(a) \phi=0.15,(b) \phi=0.18,(c) \phi=0.2$ on the stream line at $n=$ $1.1, b=0.05, k=0.1, F=0.2$
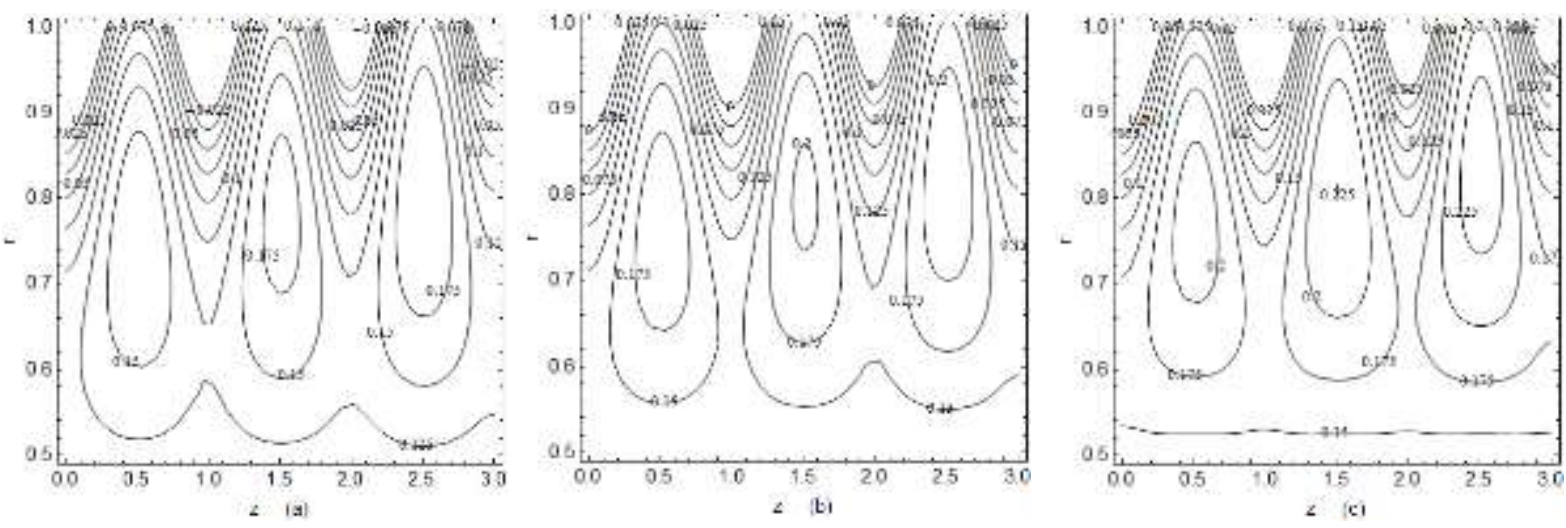

Figure 30- Effect of $(a) F=0.1,(b) F=0.15,(c) F=0.2$ on the stream line at $n=$ $1.1, b=0.05, k=0.1, \phi=0.2$

\section{Conclusions}

The present study deals with the peristaltic transport of power-law fluid in an elastic tapered tube of variable cross sections induced by dilating peristaltic wave. The biological systems deal with elastic boundaries, so that the non-Newtonian fluid flow through elastic walls gives some influential applications such as the swallowing of food through the esophagus. The presented investigation can be applicable for the patients suffering from sliding hiatus hernia, which happens when the esophagus is divided into two parts. First we consider that the esophagus gets diverged near the distal end, while the second consideration is that, at the distal end, some part of the esophagus gets diverged and then converged. The exact solutions of the problem are obtained under the assumptions of low Reynolds number and long wavelength. The influence of various parameters in the problem is explained graphically. From the above results, we concluded that the increase in $n, k$ and $\phi$ leads to an increase in the axial velocity at the center of the tube, which is decreased near the boundary. Whereas the opposite behavior is noted for $b$ which is increased with increasing $\mathrm{F}$ in all cross sections of the tube. Also, we concluded that the radial velocity distribution is parabolic in nature and it is reduced with increasing $n, k, \phi$ and $F$, it is increased with increasing $b$, and it is very sensitive to the variation of $\phi$. The volume flow rate is sinusoidal in nature and it is an increasing function with the increase of $n, k, \phi$ and $F$, whereas it is a decreasing function with the increase of $b$. It is noted that the pressure gradient decreases with the increase of $n, k, \phi$ and $\frac{\partial p}{\partial z}$, while it increases with the increase of $b$. An opposite behavior for shear strain is noticed compared to pressure gradient. The size of the trapping bolus increases with increasing $n, b, \phi$ and $F$, whereas it decreases as $k$ increases. 


\section{References}

1. Sreenadh, S., Sumalatha, B., Srinivas, A.N.S. 2018. Flow of a Herschel-Bulkley fluid in a channel with elastic walls, International Journal of Engineering \& Technoilogy, 7(4.10): 491-496.

2. Sreenadh, S. , Komala, K., Srinivas, A.N.S. 2017. Peristaltic pumping of a power-law fluid in contact with a Jeffrey fluid in an inclined channel with permeable walls, Ain Shams Engineering Journal, 8: 605-611.

3. Yang, H., Lin, J., Ku, X. 2019. Mixture flow of particles and power-law fluid in round peristaltic tube, Applied Mathematics and Mechanics (English Edition), 40: 805-822.

4. Srivastava, L.M., Srivastava, V.P. 1988. Peristaltic transport of a power-law fluid: application to the ductus efferentes of the reproductive tract, Rheol Acta, 27: 428-433.

5. Vajravelu K., Sreenadh S., Viswanatha Reddy G. 2006. Helical flow of a power-law fluid in a thin annulus with permeable walls, International Journal of Non-linear Mechanics, 41: 761-765.

6. Pandey, S.K., Chaube M.K. 2010. Peristaltic transport of a visco-elastic fluid in a tube of nonuniform cross section, Mathematical and Computer Modelling, 52: 501-514. DOI: 10.1016 /j. mcm. 2010.03.047

7. Sadeghi, K., Jalali Talab, H. 2014. Analytical investigation of peristaltic transport of power law fluid through a tube. Journal of Applied Mechanical Engineering, 3: 136. DOI: 10.4172/21689873.1000136

8. Hins, S., Mustafa, M., Hayat, T., Alsaadi, F. E. 2014. Peristaltic motion of third grade fluid in curved channel, Applied Mathematics and Mechanics (English Edition), 35(1): 73-84.

9. Vajravelu, K., Sreenadh, S., Devaki, P. 2016. peristaltic pumping of a Casson fluid in an elastic tube, Journal of Applied Fluids Mechanics, 9: 1897-1905.

10. Maiti, S., Pandey, S. K. 2017. Rheological fluid motion in tube by metachronal waves of cilia, Applied Mathematics and Mechanics (English Edition), 38(3): 393-410.

11. Selvi, C.K., Srinivas, A.N.S., Sreenadh, S. 2018. Peristaltic transport of a power-law fluid in an elastic tube, Journal of Taibah University for Science, 12(5): 687-698. DOI: 10.1080/16583655 .2018 .1503783

12. Pandey, S.K., Singh, A. 2019. Peristaltic transport of Herschel-Bulkley fluids in tubes of variable cross section induced by dilating peristaltic waves: application to sliding hiatus hernia, International Journal of Dynamics and Control, 7: 407-418.

13. Selvi, C.K., Srinivas, A.N.S. 2019. Peristaltic transport of Herschel-Bulkley fluids in a nonuniform elastic tube, Propulsion and Power Research, 8(3): 253-262.

14. Adnan, F.A. and Abdualhadi, A.M. 2019. Effect of an inclined magnetic field on peristaltic flow of Bingham plastic fluid in an inclined symmetric channel with slip conditions, Iraqi Journal of Science, 60(7): 1551-1574. DOI: 10.24996/ijs.2019.60.7.16.

15. Rabiha S. Kareem and Abdualhadi, A.M. 2020. Impacts of Heat and Mass Transfer on Magneto Hydrodynamic Peristaltic Flow Having Temperature-dependent Properties in an Inclined Channel Through Porous Media, Iraqi Journal of Science, 61(4): 854-869.DOI:10.24996/ijs.2020.61.4.19.

16. Saba S. Hasen and Abdualhadi, A.M. 2020. MHD Effect on Peristaltic Transport for Rabinowitsch Fluid through A Porous Medium in Cilia Channel, Iraqi Journal of Science, 61(6): 1461-1472. DOI: 10.24996/ijs.2020.61.6.26.

17. Sankan, G. S. and Patil, A. 2018. Heat transfer inferences on the Herschel-Bulkley fluid flow under peristalsis, Frontiers in Heat and Mass Transfer, 10(17): 1-8.

18. Michael Lai, Erhard Krempl and David Ruben, 2010. Introduction To Continuum Mechanics, fourth edition, Elsevier Inc, USA. 